Revista de Metalurgia 52(1)

Enero-Marzo 2016, e060

ISSN-L: 0034-8570

doi: http://dx.doi.org/10.3989/revmetalm.060

\title{
Influencia de la adición de estaño en el proceso de precipitación en una aleación de $\mathrm{Cu}-\mathrm{Ni}-\mathrm{Zn}$
}

\author{
Eduardo C. Donoso ${ }^{\mathrm{a}, \bowtie}, \mathrm{M}^{\mathrm{a}}$ Jesús Diánez ${ }^{\mathrm{b}}$, José M. Criado ${ }^{\mathrm{b}}$, Rodrigo Espinoza ${ }^{\mathrm{a}}$, Edgar Mosquera ${ }^{\mathrm{a}}$ \\ ${ }^{a}$ Universidad de Chile, Facultad de Ciencias Físicas y Matemáticas, Departamento de \\ Ciencia de los Materiales, Avda. Tupper 2069, Santiago de Chile, Chile \\ ${ }^{b}$ Instituto de Ciencias de Materiales de Sevilla, CSIC, Centro Mixto Universidad de \\ Sevilla-CSIC, calle Américo Vespucio 49, 41092 Sevilla, España \\ Autor para la correspondencia: edonoso@ing.uchile.cl
}

Enviado: 9 Marzo 2015; Aceptado: 26 Octubre 2015; Publicado on-Line: 2 Febrero 2016

\begin{abstract}
RESUMEN: Mediante calorimetría diferencial de barrido (DSC), medidas de microdureza y microscopía electrónica de transmisión de alta resolución (HRTEM) se estudió la influencia de la adición de 1,1\% en peso de estaño en el endurecimiento por precipitación en una aleación de $\mathrm{Cu}-11 \mathrm{Ni}-20 \mathrm{Zn}$ ( $\%$ en peso). Las curvas calorimétricas de la aleación ternaria, en el intervalo de temperaturas analizado, muestran la presencia de dos reacciones exotérmicas asociadas al desarrollo de orden de corto alcance, favorecidas por la migración de vacantes. En cambio, en la aleación cuaternaria se observa una reacción exotérmica y una endotérmica, asociadas a la formación y disolución de precipitados de $\mathrm{Cu}_{2} \mathrm{NiZn}$, respectivamente. Se ha demostrado que una adición de $1,1 \%$ de estaño juega un papel importante en la formación de los precipitados de $\mathrm{Cu}_{2} \mathrm{NiZn}$, responsables del endurecimiento por precipitación de la aleación ternaria.
\end{abstract}

PALABRAS CLAVE: Aleaciones de Cu-Ni-Zn; Aleaciones de Cu-Ni-Zn-Sn; Calorimetría; Cinética; Endurecimiento por precipitación

Citation / Cómo citar este artículo: Donoso, E.C., Diánez, M.J., Criado, J.M., Espinoza, R., Mosquera, E. (2016) "Influencia de la adición de estaño en el proceso de precipitación en una aleación de Cu-Ni-Zn". Rev. Metal. 52(1):e060. doi: http://dx.doi.org/10.3989/revmetalm.060.

ABSTRACT: Influence of tin additions on the precipitation processes in a $\mathrm{Cu}$-Ni-Zn alloys. The influence of $1.1 \mathrm{wt} \%$ tin additions on the precipitation hardening of $\mathrm{Cu}-11 \mathrm{wt} \% \mathrm{Ni}-20 \mathrm{wt} \% \mathrm{Zn}$ alloy was studied by Differential Scanning Calorimetry (DSC), microhardeness measurements and High Resolution Transmission Electron Microscopy (HRTEM). The calorimetric curves, in the range of temperatures analyzed, show the presence of two exothermic reactions in the ternary alloy, associated to the short-range-order development assisted by migration of excess vacancies. On the other hand, one exothermic and one endothermic reaction are observed in the quaternary alloy, associated to the formation and dissolution of $\mathrm{Cu}_{2} \mathrm{NiZn}$ precipitates, respectively. It has been show that an addition of $1.1 \%$ tin plays an important role in the formation of $\mathrm{Cu}_{2} \mathrm{NiZn}$ precipitates, responsible for the precipitation hardening of the ternary alloy.

KEYWORDS: Calorimetry; Cu-Ni-Zn alloys; $\mathrm{Cu}-\mathrm{Ni}-\mathrm{Zn}-\mathrm{Sn}$ alloys; Kinetic; Precipitation hardening

Copyright: (C) 2016 CSIC. This is an open-access article distributed under the terms of the Creative Commons Attribution-Non Commercial (by-nc) Spain 3.0 License. 


\section{INTRODUCCIÓN}

La industria naval y aeroespacial demanda nuevos materiales microestructuralmente estables dotados de una elevada resistencia mecánica a la vez que una alta conductividad eléctrica y/o térmica y una gran resistencia a la corrosión (Groza y Gibeling, 1993). El cobre es el mejor candidato para estas aplicaciones debido a su alta resistencia a la corrosión unida a sus elevadas conductividades eléctrica y térmica. No obstante, presenta el hándicap de una baja resistencia mecánica, por lo que es necesario mejorar estas propiedades, bien mediante la dispersión de pequeñas fracciones de materiales cerámicos como alúmina o carburo de titanio (Palma et al., 2004; Palma et al., 2005; Palma et al., 2010), o bien mediante aleado con otros metales (Nagarjuna et al., 2000; Nagarjuna et al., 2006; Zhou et al., 2010).

En general, las aleaciones de $\mathrm{Cu}-\mathrm{Ni}-\mathrm{Zn}$ (con composiciones entre $5-20 \% \mathrm{Ni}$ y $5-30 \% \mathrm{Zn}$ ) son las utilizadas con más frecuencia para las aplicaciones antes indicadas (Lebrun y Perrot, 2007; Zhou et al., 2010), dado que la adición de níquel mejora considerablemente las propiedades mecánicas mientras que el estaño contribuye a disminuir tanto el punto de fusión como la porosidad. El método de endurecimiento por refinamiento de grano por laminación ha sido el más empleado hasta ahora para incrementar la resistencia mecánica de estas aleaciones (Nagarjuna et al., 2000; Nagarjuna et al., 2006). Sin embargo, el incremento de las microtensiones por un severo laminado en frío presenta el inconveniente de llevar asociada una disminución de la conductividad eléctrica (Nagarjuna et al., 2006; Zhou et al., 2010), mientras que, por el contrario, si el fortalecimiento de las aleaciones se produce por precipitación tiene lugar un aumento simultáneo de la tenacidad y de la conductividad eléctrica (Zhou et al., 2010). Puesto que, de acuerdo con el diagrama de fases de esta aleación ternaria (De Rooy et al., 1981, Van der Wegen et al., 1981), el $\mathrm{Cu}_{2} \mathrm{NiZn}$ es la única fase susceptible de segregarse por precipitación, es de interés tener en cuenta sus características estructurales para poder interpretar las modificaciones sufridas por las propiedades mecánicas durante el recocido de aleaciones de $\mathrm{Cu}-\mathrm{Ni}-\mathrm{Zn}$. Los cambios estructurales experimentados por el $\mathrm{Cu}_{2} \mathrm{NiZn}$ en función de la temperatura (Van der Wegen et al., 1983; Simak et al., 1998) pueden explicarse dividiendo la estructura cúbica centrada en caras (fcc) en cuatro sub-retículos cúbicos interpenetrados entre sí con las siguientes coordenadas: I (000), II $(1 / 2,1 / 2,1 / 2)$, III $(1 / 2,0,1 / 2)$ y IV $(0,1 / 2,1 / 2)$. En la fase completamente ordenada $\mathrm{Ll}_{0}$ (tipo $\mathrm{AuCu}$ ), estable hasta 600 K (Hirabayashi et al., 1965; Ito y Nakayama, 1988; Althqff y Johnson, 1997; Lebrun y Perrot, 2007), dichas posiciones reticulares están ocupadas por $\mathrm{Zn}$, Ni y $\mathrm{Cu}$, respectivamente. A temperaturas superiores a $600 \mathrm{~K}$, se produce una transición a la fase $\mathrm{Ll}_{2}$ parcialmente ordenada (tipo $\mathrm{AuCu}_{3}$ ) en la que los átomos de zinc se posicionan en los vértices del cubo mientras que los átomos de cobre y níquel están distribuidos al azar en los sub-retículos II, III y IV. Puesto que las aleaciones de Cu-Ni-Zn con porcentajes de zinc inferiores al 30\% forman soluciones sólidas estables en todo el rango de composición (Lebrun y Perrot, 2007; Zhou et al., 2010) se ha sugerido (Zhou et al., 2010) que la adición de un pequeño porcentaje de un cuarto elemento podría propiciar el endurecimiento de la aleación por precipitación. Se ha descrito recientemente en la bibliografía (Zhou et al., 2010) que la adición de aluminio en concentraciones en torno al 1\% conduce a un extraordinario aumento de la microdureza de la aleación debido a la formación de precipitados de $\mathrm{Cu}_{2} \mathrm{NiZn}$. La influencia de la adición de pequeñas cantidades de otros metales distintos del aluminio en el endurecimiento por precipitación de aleaciones $\mathrm{Cu}-\mathrm{Ni}-\mathrm{Zn}$ no ha sido aún estudiada. El estaño podría ser un candidato idóneo para este propósito habida cuenta de que contribuiría a disminuir el punto de fusión de la aleación. La única publicación referida a aleaciones cuaternarias Cu-Ni-Zn-Sn (Wnuk y Zielinska, 2009) está dirigida al estudio de la temperatura de cristalización de aleaciones fundidas de distinta composición, así como a la determinación de las entalpías de fusión de las citadas aleaciones, sin incluir ningún estudio de la influencia del recocido en sus propiedades mecánicas.

El presente trabajo aborda por primera vez el estudio del mecanismo de endurecimiento por precipitación de una aleación de $\mathrm{Cu}-11 \mathrm{Ni}-20 \mathrm{Zn}(\%$ en peso) cuando se adicionan pequeñas concentraciones de estaño.

\section{MATERIALES Y MÉTODOS}

Las aleaciones utilizadas se prepararon en un horno de inducción, en atmósfera inerte (Ar), a partir de las cantidades estequiométricas de cobre electrolítico (99,95\% de pureza), níquel, cinc y estaño de alta pureza para obtener la composición de la aleación deseada. Los lingotes obtenidos se recocieron a $1123 \mathrm{~K}$ durante 24 horas (en atmósfera inerte) a fin de homogeneizarlos y se analizó la composición química de los mismos por fluorescencia de rayos X (FRX). Los porcentajes en peso de los elementos contenidos en la aleación de composición nominal $\mathrm{Cu}-11 \% \mathrm{Ni}-20 \% \mathrm{Zn}$ fueron $\mathrm{Cu}-11,3 \% \mathrm{Ni}-19,9 \% \mathrm{Zn}$, mientras que los de la aleación de composición nominal $\mathrm{Cu}-11 \% \mathrm{Ni}-19 \% \mathrm{Zn}-1 \% \mathrm{Sn}$ responden a la composición real $\mathrm{Cu}-11,3 \%-\mathrm{Ni}-19,2 \% \mathrm{Zn}-1,1 \% \mathrm{Sn}$. Posteriormente, los materiales se laminaron en frío desde $6 \mathrm{~mm}$ hasta $3 \mathrm{~mm}$ de espesor con recocidos intermedios de 1 hora a $1123 \mathrm{~K}$ con objeto de homogeneizar las muestras. Después del último recocido, las aleaciones se templaron en agua. 
Para el análisis calorimétrico se ha utilizado un equipo de calorimetría diferencial de barrido (DSC) TA Instruments Q10. Los diagramas de DSC se han registrado a diferentes velocidades de calentamiento $\left(\beta=5,10,20,30\right.$ y $\left.40 \mathrm{~K} \mathrm{~min}^{-1}\right)$, bajo un flujo de argón de $10^{-4} \mathrm{~m}^{3} \mathrm{~min}^{-1}$, utilizando como referencia un disco de cobre de alta pureza recocido durante un largo período. La corrección de la línea base se llevó a cabo por el método descrito previamente por Varchavsky y Donoso (2002), Varchavsky y Donoso (2003) y Donoso et al. (2007). Los valores de $\alpha$ a cada temperatura se calcularon a partir del cociente entre el área de la curva de DSC hasta la temperatura considerada y el área total de la curva siguiendo el método convencional descrito en trabajos anteriores (Donoso, 2010; Donoso, 2014).

Las medidas de microdureza Vickers se efectuaron a temperatura ambiente en un microdurómetro Duramin -1/-2 Struers de alta precisión, aplicando una carga de 1,96 $\mathrm{N}$ durante 10 segundos. Cada valor de microdureza corresponde al promedio de diez medidas, con una desviación estándar de aproximadamente un $2 \%$. La observación por microscopía TEM se realizó en un microscopio electrónico de transmisión, Philips CM-200 con una tensión de trabajo de $200 \mathrm{kV}$ y un filamento de $\mathrm{LaB}_{6}$.

Los diagramas de difracción de rayos $\mathrm{X}$ (XRD) se registraron con un difractómetro PHILIPS X'pert Pro equipado con un detector de centelleo y un monocromador de grafito empleando un voltaje de $40 \mathrm{kV}$ y $30 \mathrm{~mA}$.

\section{RESULTADOS Y DISCUSION}

La Fig. 1 muestra curvas calorimétricas típicas registradas a una velocidad de calentamiento lineal $\beta=10 \mathrm{~K} \mathrm{~min}^{-1}$ para las dos aleaciones en estudio: (a) $\mathrm{Cu}-10,9 \% \mathrm{Ni}-19,9 \% \mathrm{Zn}$ (tomada como referencia)

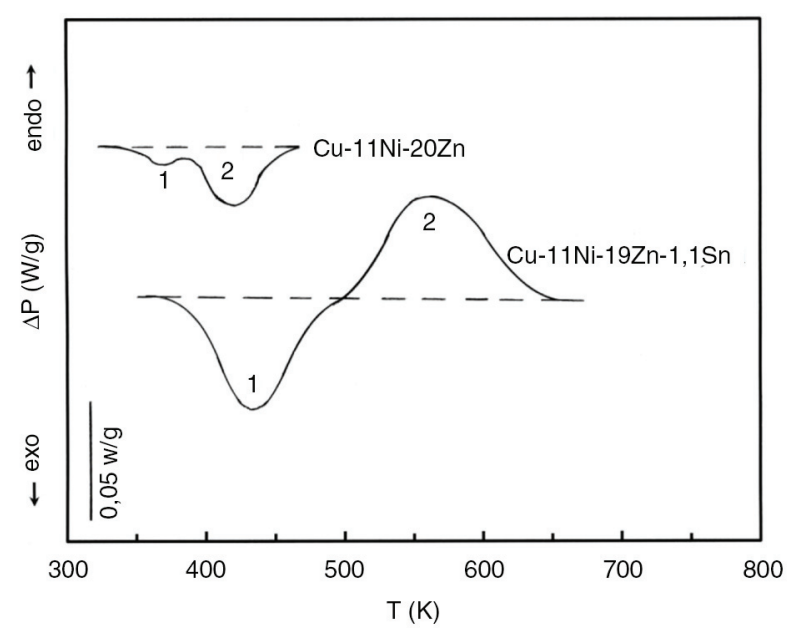

Figura 1. Curvas DSC de las aleaciones en estudio templadas desde $1123 \mathrm{~K} . \beta=10 \mathrm{~K} \mathrm{~min}^{-1}$

a) $\mathrm{Cu}-10,9 \% \mathrm{Ni}-19,9 \% \mathrm{Zn}$ y b) $\mathrm{Cu}-11,3 \% \mathrm{Ni}-19,2 \% \mathrm{Zn}-1,1 \% \mathrm{Sn}$. y (b) $\mathrm{Cu}-11,3 \% \mathrm{Ni}-19,2 \% \mathrm{Zn}-1,1 \% \mathrm{Sn}$, previamente templadas desde $1123 \mathrm{~K}$. Las curvas calorimétricas para las otras velocidades de calentamiento lineal tienen un comportamiento similar al mostrado en la Fig. 1 y no se muestran en este trabajo, aunque las temperaturas de los picos de la aleación cuaternaria obtenidos a distintas velocidades de calentamiento, que se utilizarán más adelante para el estudio cinético, se incluyen en la Tabla 1. El hecho de que las temperaturas de los picos de las curvas calorimétricas se desplacen a temperaturas más altas al aumentar la velocidad de calentamiento pone de manifiesto el control cinético del proceso (Varschavsky y Donoso, 1991).

Las curvas DSC del $\mathrm{Cu}-10,9 \% \mathrm{Ni}-19,9 \% \mathrm{Zn}$, registradas en el intervalo $25-950 \mathrm{~K}$, muestran a temperaturas inferiores a $500 \mathrm{~K}$ dos etapas exotérmicas solapadas. Teniendo en cuenta el diagrama de fases de la aleación ternaria $\mathrm{Cu}-\mathrm{Ni}-\mathrm{Zn}$ mencionado en la introducción, el primer pico exotérmico podría estar asociado a la formación de la fase $\mathrm{Ll}_{0}$, y el segundo pico correspondería a la transición de fase $\mathrm{Ll}_{0} \rightarrow \mathrm{Ll}_{2}$. Es importante indicar que el análisis de esta aleación por microscopía electrónica de transmisión realizado en un trabajo anterior (Donoso et al., 2014) ha puesto de manifiesto que dichas fases están integradas como fases coherentes en la matriz de cobre $\alpha$ sin formar precipitados. Este comportamiento será de interés para interpretar las propiedades mecánicas que se analizarán más adelante.

El diagrama de DSC de la aleación cuaternaria con un contenido en estaño del 1,1\% que se muestra en la Fig. 1 pone de manifiesto que el doble pico exotérmico observado para la aleación ternaria se convierte en la aleación cuaternaria en un único pico exotérmico en torno a los $480 \mathrm{~K}$, aunque con una entalpía muy superior a la de la aleación ternaria si se considera el área del pico correspondiente de DSC. Este comportamiento podría atribuirse a la formación de precipitados de $\mathrm{Cu}_{2} \mathrm{NiZn}$ promovida por la adición de estaño. El pico endotérmico que se observa a $550 \mathrm{~K}$ podría corresponder a la redisolución de dichos precipitados al elevar la temperatura.

Con el objeto de analizar la posible relación existente entre los efectos térmicos observados por DSC y las propiedades mecánicas de las aleaciones estudiadas, se han realizado medidas de microdureza Vickers en función del tiempo de recocido a distintas temperaturas comprendidas en el intervalo de temperaturas en el que se observan los picos en los diagramas mencionados. Con este propósito se calentaron una serie de muestras en el equipo de DSC a una velocidad de calentamiento de $10 \mathrm{~K} \mathrm{~min}^{-1}$ y se extrajeron del equipo al alcanzarse las temperaturas de 450,525 y $675 \mathrm{~K}$, respectivamente, y posteriormente se midió la dureza en función del tiempo de recocido para cada una de las muestras a la misma temperatura a la que se interrumpió el experimento de DSC. 
TABLA 1. Temperatura de los picos y parámetros cinéticos obtenidos a partir de los diagramas de DSC de la aleación de composición Cu-11,3\%Ni-19,2\%Zn-1,1\%Sn

\begin{tabular}{|c|c|c|c|c|c|}
\hline & 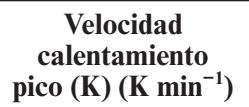 & Temperatura & $\begin{array}{c}E \\
\left(\mathrm{~kJ} \mathrm{~mol}^{-1}\right)\end{array}$ & $\ln \mathrm{A}^{\mathrm{a}}(n)$ & Coeficiente $^{b}$ \\
\hline \multirow{5}{*}{$\begin{array}{l}\text { Pico } 1 \\
\text { (Exotérmico) }\end{array}$} & 5 & 420 & & & \\
\hline & 10 & 430 & & & \\
\hline & 20 & 450 & $148 \pm 2$ & $20,5 \pm 0,5$ & $1,47 \pm 0,06$ \\
\hline & 30 & 470 & & & \\
\hline & 40 & 485 & & & \\
\hline \multirow{5}{*}{$\begin{array}{l}\text { Pico } 2 \\
\text { (Endotérmico) }\end{array}$} & 5 & 560 & & & \\
\hline & 10 & 575 & & & \\
\hline & 20 & 600 & $194 \pm 3$ & $23,7 \pm 0,5$ & $*$ \\
\hline & 30 & 605 & & & \\
\hline & 40 & 610 & & & \\
\hline
\end{tabular}

${ }^{\mathrm{a}} \mathrm{A}$ en s ${ }^{-1}$; ${ }^{\mathrm{b}}$ Coeficiente de Avrami-Erofeev; ${ }^{*}$ Este proceso se ajusta a un modelo de reacción controlado por el avance de la interfase y no a un mecanismo de Avrami-Erofeev.

La Fig. 2 muestra la variación de la microdureza en función del tiempo de envejecimiento de la aleación $\mathrm{Cu}-11,3 \%-\mathrm{Ni}-19,2 \mathrm{Zn}-1,1 \% \mathrm{Sn}$ a temperaturas de recocido de 450, 525 y $675 \mathrm{~K}$; también se incluye en la misma figura, a título comparativo, la variación de la dureza de la aleación ternaria $\mathrm{Cu}-10,9 \% \mathrm{Ni}-19,9 \% \mathrm{Zn}$ en función del tiempo de recocido a $450 \mathrm{~K}$. Los valores de microdureza obtenidos muestran claramente que la adición de estaño a la aleación de $\mathrm{Cu}-\mathrm{Ni}-\mathrm{Zn}$ incrementa el endurecimiento de la matriz de 86 a $126 \mathrm{HV}$ al templar el material, debido principalmente al cambio de parámetro de red de la aleación (Pearson, 1958). Sin embargo, cuando estas aleaciones se someten a tratamientos de envejecimiento, la aleación ternaria alcanza un máximo de $102 \mathrm{HV}$ tras un recocido a $450 \mathrm{~K}$ durante 240 minutos, mientras que la aleación con estaño alcanza una microdureza de 186 HV cuando el material es recocido durante

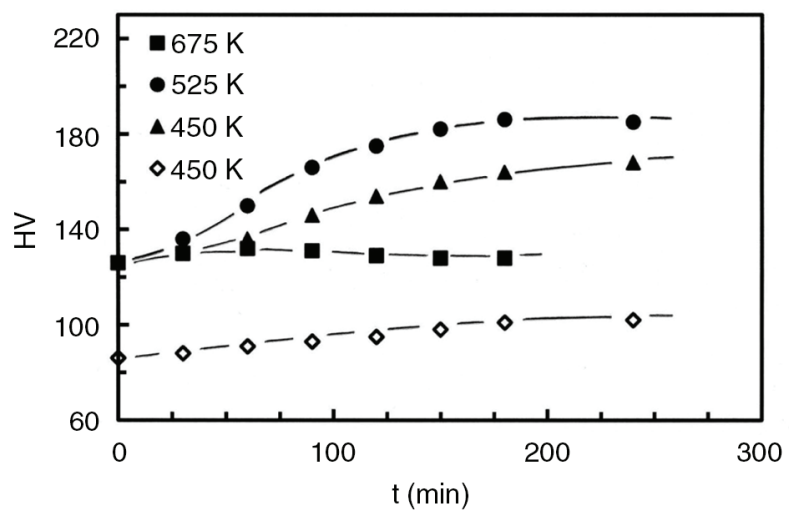

Figura 2. Medidas de microdureza Vickers versus tiempo a varias temperaturas de envejecimiento (las muestras han sido previamente templadas desde $1123 \mathrm{~K}$ ).

( $) \mathrm{Cu}-10,9 \% \mathrm{Ni}-19,9 \% \mathrm{Zn},(\mathbf{\bullet}, \bullet, \mathbf{\Delta}) \mathrm{Cu}-11,3 \% \mathrm{Ni}-19,2 \% \mathrm{Zn}-1,1 \% \mathrm{Sn}$
180 minutos a la temperatura de $525 \mathrm{~K}$, asociada al pico exotérmico. Este hecho sugiere que el pico exotérmico observado en el diagrama de DSC de la aleación cuaternaria está asociado a la formación de una fase $\mathrm{Cu}_{2} \mathrm{NiZn}$ de modo análogo al comportamiento observado al añadir aluminio a una aleación de Cu-Ni-Zn (Zhou et al., 2010) Por otra parte, se observa una fuerte disminución de la microdureza al recocer la aleación Cu-11,3\%-Ni-19,2Zn-1,1\%Sn a la temperatura de $675 \mathrm{~K}$, asociada al pico endotérmico, lo que apoya el atribuir este pico a la redisolución de los precipitados de $\mathrm{Cu}_{2} \mathrm{NiZn}$ previamente formados en el intervalo de temperaturas en que se observa el efecto exotérmico.

Con el objeto de confirmar la formación de precipitados se realizó un análisis por microscopía electrónica de alta resolución (HRTEM) de la aleación $\mathrm{Cu}-11,3 \%-\mathrm{Ni}-19,2 \mathrm{Zn}-1,1 \% \mathrm{Sn}$ templada desde $1123 \mathrm{~K}$ y recocida a $525 \mathrm{~K}$ durante 180 minutos. Las micrografías permiten observar partículas esféricas coherentes de tamaño nanométrico $(4 \mathrm{~nm}$ de promedio) distribuidas homogéneamente en la matriz de cobre, como se pone de manifiesto en la Fig. 3. Partículas similares se observaron en una aleación de Cu-10Ni-20Zn-1.2Al recocida a $723 \mathrm{~K}$ (Zhou et al., 2010). El hecho de que estos precipitados no se observen en las microfotografías de la muestra de la aleación cuaternaria recocida a $675 \mathrm{~K}$ sugiere la asociación del pico endotérmico a la disolución de los precipitados de $\mathrm{Cu}_{2} \mathrm{NiZn}$ presumiblemente formados en la etapa anterior. Desafortunadamente no se ha podido determinar por EDX la composición de los precipitados debido a que el área mínima cubierta por el Microscopio TEM utilizado es muy superior al tamaño de los precipitados ( $\approx 4 \mathrm{~nm})$. Por la misma razón no se ha podido poner de manifiesto la presencia de dichos precipitados por difracción 


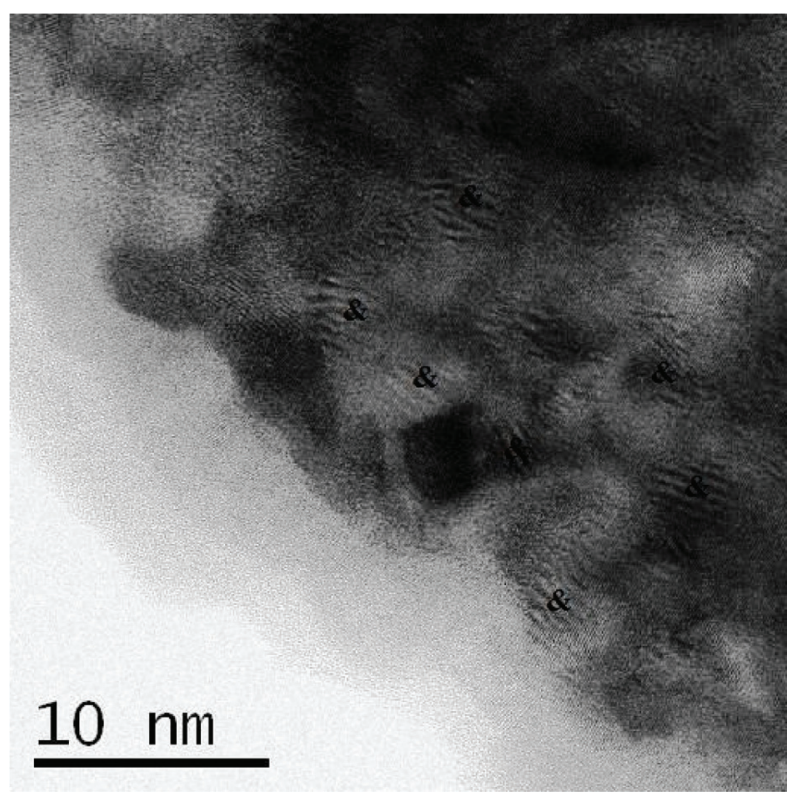

Figura 3. Micrografia HRTEM de la aleación $\mathrm{Cu}-11,3 \% \mathrm{Ni}-19,2 \% \mathrm{Zn}-1,1 \% \mathrm{Sn}$ recocida a $525 \mathrm{~K}$. (\&) Nanocristales de $\mathrm{Cu}_{2} \mathrm{NiZn}$.

de rayos $\mathrm{X}$ según puede observarse en la Fig. 4 en la que se comparan los diagramas de difracción de rayos $\mathrm{X}$ de la muestra $\mathrm{Cu}-11,3 \%-\mathrm{Ni}-19,2 \mathrm{Zn}-1,1 \% \mathrm{Sn}$ templada y recocida a $525 \mathrm{~K}$, respectivamente. Estos resultados ponen de manifiesto que solo se observan en ambos casos los picos de difracción correspondiente a la fase rica en cobre característica de la disolución sólida que constituye la aleación. Este comportamiento puede explicarse si se tiene en cuenta que se requerirían tamaños de partículas muy superiores a $4 \mathrm{~nm}$ para que la anchura de los picos de DRX esté por debajo del umbral crítico para su discriminación de la línea base del diagrama.

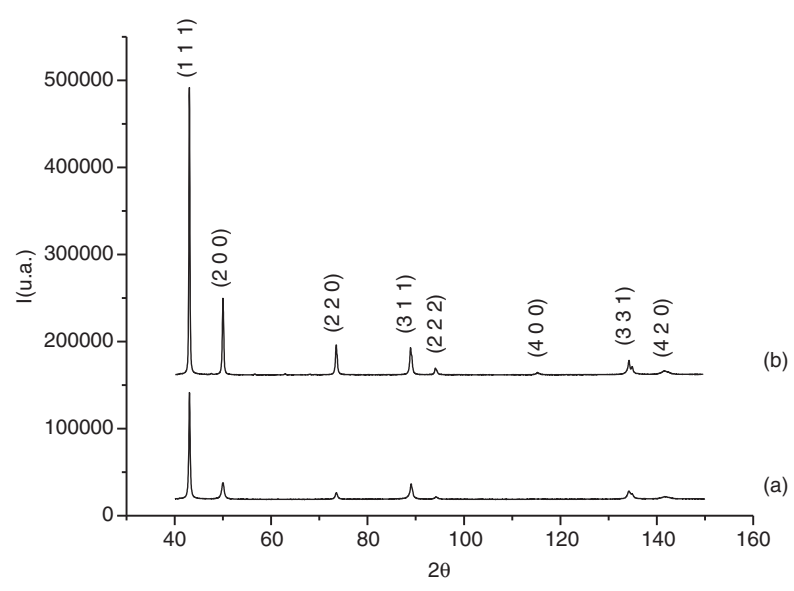

Figura 4. Diagramas de difracción de rayos $\mathrm{X}$ de la muestra Cu-11,3\% Ni-19,2\% Zn-1,1\% Sn: a) Muestra templada y b) Muestra recocida a $525 \mathrm{~K}$.
Es bien conocido en la bibliografía (Christian, 2002; Svadlak et al., 2008) que tanto los procesos de cristalización como los que involucran transiciones de fase en estado sólido están gobernados por un mecanismo que implica la formación y crecimiento de núcleos representado por el modelo cinético de Avrami-Erofeev, expresado por la siguiente ecuación cinética:

$\alpha=1-\exp \left[-(A \theta)^{n}\right]$

donde $\alpha$ es la conversión a la temperatura $T, A$ es el factor pre-exponencial de Arrhenius y $\theta$ es el tiempo reducido descrito por la siguiente expresión:

$\theta=\frac{R T^{2}}{\beta E} \exp \left(-\frac{E}{R T}\right)$

siendo $E$ la energía de activación, $\beta$ la velocidad de calentamiento lineal y $R$ la constante de los gases (Ozawa, 1976).

La Ec. (1) es válida para el análisis cinético de datos experimentales obtenidos tanto en régimen isotermo como en programa lineal de calentamiento (Gotor et al., 2000) y puede ser representada en su forma logarítmica por la siguiente expresión:

$\ln [-\ln (1-\alpha)]=n \ln A+n \ln \theta$

El coeficiente $n$ de Avrami-Erofeev y el factor pre-exponencial de Arrhenius, $A$, pueden determinarse a partir de la línea recta resultante de la representación gráfica del primer miembro de la Ec. (3) en función de $\ln \theta$. La determinación del valor de $\theta$ a cada temperatura a partir de la Ec. (2) implica un conocimiento previo de la energía de activación que requiere su determinación por un procedimiento independiente. Un análisis más detallado del desarrollo de las ecuaciones cinéticas anteriormente descritas se ha incluido en una publicación anterior (Donoso et al., 2012).

Las energías de activación se han determinado a partir del método de Kissinger (Kissinger, 1957) que no requiere un conocimiento previo del mecanismo de reacción para determinar dicho parámetro a partir de las temperaturas de los picos de DSC obtenidos a diferentes velocidades de calentamiento mediante la siguiente ecuación cinética:

$\ln \left(\frac{T_{p}^{2}}{\beta}\right)=\left(\frac{E}{R}\right) \frac{1}{T_{p}}+C$

siendo $T_{p}$ la temperatura del máximo del pico de DSC y $C$ una constante. La representación del primer miembro de la Ec. (4) en función de la inversa de $T_{\mathrm{p}}$ sería una línea recta de cuya pendiente se obtendría la energía de activación del proceso. 
Por otra parte, Mittenmeijer (Mittemeijer et al., 1988) ha puesto de manifiesto que en el caso particular de que la reacción se ajuste a un modelo de Avrami-Erofeev, la ecuación de Kissinger podría escribirse del siguiente modo:

$\ln \left(\frac{T_{p}^{2}}{\beta}\right)=\left(\frac{E}{R}\right) \frac{1}{T_{p}}+\ln \left(\frac{E}{A R}\right)$

donde $E$ y $A$ podrían determinarse a partir de la pendiente y de la ordenada en el origen de la línea recta resultante de la representación gráfica de los valores de $\ln \left(T_{p}^{2} / \beta\right)$ frente a $1 / T_{p}$, de acuerdo con la Ec. (5). La Tabla 1 muestra los valores de $E, A$ y $n$ obtenidos del proceso exotérmico reportado en el diagrama de DSC de la aleación Cu-11,3\%-Ni-19,2Zn-1,1\%Sn. Los valores de $A$ y $n$ se han calculado mediante el análisis combinado de las Ecs. (3) y (5) tras determinar la energía de activación a partir de los datos de $T_{\mathrm{p}}$ obtenidos a distintas velocidades de calentamiento reportados en la Tabla 1. Es importante señalar que el factor pre-exponencial de Arrhenius determinado a partir de la Ec. (3) muestra un excelente acuerdo con el valor calculado mediante la Ec. (5), lo que apoya la suposición previa de que la reacción tiene lugar a través de un mecanismo de Avrami-Erofeev.

El valor del coeficiente $n$ de Avrami-Erofeev es muy próximo a 1,5 que es el valor estimado para procesos que tienen lugar mediante un mecanismo que implica el crecimiento de núcleos de tamaño nanométrico mediante un proceso de difusión (Chvoj et al., 1991). En consecuencia, este resultado apoyaría la interpretación de que la generación de los precipitados de $\mathrm{Cu}_{2} \mathrm{NiZn}$ tiene lugar a través del crecimiento de núcleos de la fase $\mathrm{Ll}_{2}$ coherentes con la matriz de estructura cobre $\alpha$. Por otra parte, el hecho de que la energía de activación reportada en la Tabla 1 para el proceso exotérmico sea muy inferior a las energías de difusión de $\mathrm{Ni}$ en $\mathrm{Cu}\left(204 \mathrm{~kJ} \mathrm{~mol}^{-1}\right), \mathrm{Zn}$ en $\mathrm{Cu}$ $\left(185 \mathrm{~kJ} \mathrm{~mol}^{-1}\right)$ y $\mathrm{Sn}$ en $\mathrm{Cu}\left(196 \mathrm{~kJ} \mathrm{~mol}^{-1}\right)($ Brown y Ashby, 1980) podría atribuirse tanto a la influencia de las dislocaciones que puedan generarse durante la formación de precipitados como a la contribución de las vacantes generadas durante el temple (Varchavsky y Donoso, 1991; Varchavsky y Donoso, 2003; Donoso et al., 2005).

La energía de activación del proceso endotérmico que se observa en el diagrama de DSC de la aleación $\mathrm{Cu}-11,3 \%-\mathrm{Ni}-19,2 \mathrm{Zn}-1,1 \% \mathrm{Sn}$ incluido en la Fig. 1 (pico 2) se ha calculado por el método de Kissinger a partir de las temperaturas, $T_{\mathrm{p}}$, de los picos registrados a distintas velocidades de calentamiento incluidos en la Tabla 1. El valor obtenido para dicho parámetro se incluye también en la misma tabla. El valor de la energía de activación obtenido se ha sustituido en la Ec. (2) con objeto de determinar los valores de $\theta$ requeridos para efectuar el análisis cinético mediante la Ec. (3). El análisis efectuado de la representación gráfica del primer miembro de dicha ecuación en función de $\ln \theta$ puso de manifiesto que no existe una correlación lineal entre ambos parámetros lo que indica que este proceso endotérmico no obedece un modelo cinético de Avrami-Erofeev, como sería de esperar para un proceso de precipitación. Este comportamiento apoya la interpretación de los resultados discutidos anteriormente que llevaron a sugerir que la etapa endotérmica podría estar asociada a la redisolución en la matriz de la aleación de los precipitados de $\mathrm{Cu}_{2} \mathrm{NiZn}$ previamente formados en la etapa exotérmica.

Se ha establecido en la bibliografía (Varschavsky y Donoso, 2002) que la redisolución de los precipitados en fase sólida es proporcional al área de la interfase de dichos precipitados. En el caso de que la velocidad sea controlada por el avance de la interfase (considerando partículas tridimensionales), el proceso estaría gobernado por la siguiente ecuación cinética:

$g(\alpha)=1-(1-\alpha)^{\frac{2}{3}} A \theta$

Si el proceso estudiado se ajustase a dicha ecuación cinética, la representación gráfica del primer miembro de la Ec. (6) en función de $\theta$ conduciría a una línea recta cuya pendiente sería el factor pre-exponencial de Arrhenius.

La Fig. 5 muestra la representación gráfica del primer miembro de la Ec. (6) frente a los correspondientes valores de $\theta$ calculados a partir del efecto endotérmico observado en el DSC de la aleación cuaternaria que se incluye en la Fig. 1. Estos resultados ponen de manifiesto el excelente ajuste de los datos experimentales a este modelo, dando lugar a una línea recta de cuya pendiente se ha obtenido el valor del factor pre-exponencial de Arrhenius que se incluye en la Tabla 1. En consecuencia, los resultados obtenidos indican que la redisolución de los

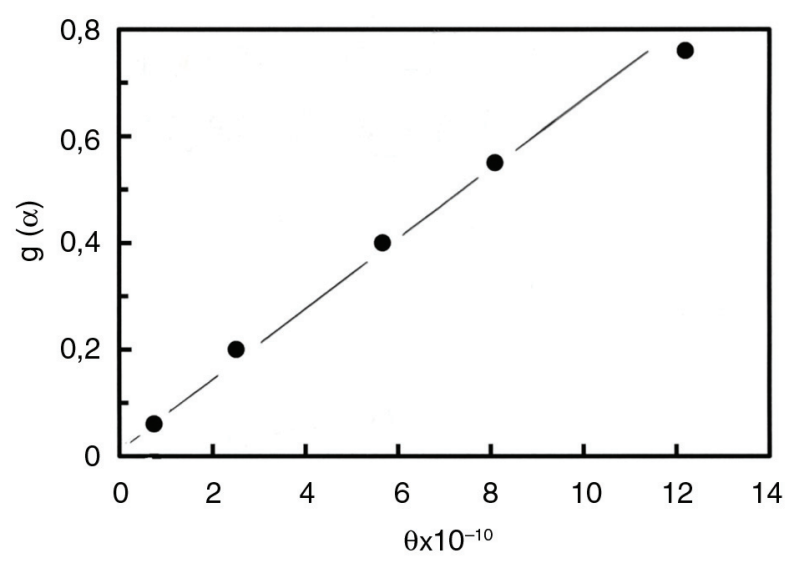

Figura 5. Análisis cinético del efecto endotérmico de la aleación Cu-11,3\%Ni-19,2\%Zn-1,1\% Sn mediante la Ec. (6). 
precipitados de $\mathrm{Cu}_{2} \mathrm{NiZn}$ tiene lugar a través de un mecanismo que implica el avance de la interfase en partículas tridimensionales. Por último, es necesario tener en cuenta que la energía de activación de $194 \mathrm{~kJ} \mathrm{~mol}^{-1}$ obtenida para este proceso coincide aproximadamente con las energías de difusión intermetálica entre los distintos elementos que componen la reacción. En consecuencia, si se considera que las energías de enlace metal-metal son próximas a las energía de difusión (Luo, 2015) puede concluirse que la ruptura de los enlaces intermetálicos controlan la velocidad de redisolución de los precipitados.

\section{CONCLUSIONES}

El análisis cinético y calorimétrico de los diagramas DSC, junto con las determinaciones de microdureza realizadas a las muestras, HRTEM y los antecedentes establecidos en la literatura permiten concluir que la adición de una pequeña concentración de estaño a una aleación de $\mathrm{Cu}-\mathrm{Ni}-\mathrm{Zn}$ conduce a un aumento del $48 \%$ en la dureza de la aleación recocida con respecto a la de la aleación templada. Este comportamiento se atribuye a la formación de precipitados de $\mathrm{Cu}_{2} \mathrm{NiZn}$ generados mediante un mecanismo de formación y crecimiento de núcleos que se ajusta a un modelo de Avrami-Erofeev.

Por otra parte, se ha puesto de manifiesto que si, tras la formación de los precipitados, se continúa incrementando la temperatura de la aleación se produce la redisolución de los mismos, con la consiguiente degradación de las propiedades mecánicas.

\section{AGRADECIMIENTOS}

Los autores agradecen al Fondo Nacional de Desarrollo Científico y Tecnológico, FONDECYT, Proyecto $\mathrm{N}^{\circ} 1140782$, por el apoyo financiero, al Instituto de Ciencia de Materiales de Sevilla y al Departamento de Ciencia de los Materiales, Facultad de Ciencias Físicas y Matemáticas, Universidad de Chile, por las facilidades otorgadas para desarrollar esta investigación.

\section{REFERENCIAS}

Althqff, J.D., Johnson, D.D. (1997). The electronic origins of atomic short-range order in disordered fcc $\mathrm{Cu}-\mathrm{Ni}-\mathrm{Zn}$ ternary alloys. J. Phase Equilib. 18 (6), 567-572. http://dx.doi.org/ 10.1007/BF02665813.

Brown, A.M., Ashby, M.F. (1980). Correlation for diffusion constants. Acta Metall. 28 (8), 1085-1101. http://dx.doi. org/10.1016/0001-6160(80)90092-9.

Christian, J.W. (2002). The Theory of Transformations in Metals and Alloys, $3^{\mathrm{a}}$ Ed., Pergamon Press, pp. 529-552.

Chvoj, Z., Sestak, J., Triska, A. (1991). Kinetic Phase Diagrams, Non Equilibrium Phase Transitions, Elsevier, Holanda, p. 222.

De Rooy, A., Van Der Wegen, G.J.L., Bronsweld, P.M., De Hosson, J.T.M. (1981). The quasi-binary cross section in the ternary system $\mathrm{Cu}-\mathrm{Ni}-\mathrm{Zn}$. II. Electrical Resistivity measurements. Scripta Metall. 15 (12), 1362-1364. http:// dx.doi.org/10.1016/0036-9748(81)90099-5.
Donoso, E., Díaz, G., Varchavsky, A. (2005). Thermal behavior of short range-order in quenched $\mathrm{Cy}-12$ at. $\% \mathrm{Mn}$ assessed by DSC. J. Therm. Anal. Calorim. 81 (2), 425-433. http:// dx.doi.org/10.1007/s10973-005-0802-7.

Donoso, E., Diánez, M.J., Sayagués, M.J., Criado, J.M., Varschavsky, A., Díaz, G. (2007). Non isothermal calorimetric study of the precipitation processes in a Cu- $10 \% \mathrm{Ni}-3 \% \mathrm{Al}$. Rev. Metal. 43 (2), 117-124. http://dx.doi.org/10.3989/ revmetalm.2007.v43.i2

Donoso, E. (2010). Influencia de la adición de cobalto y cromo en el proceso de precipitación en una aleación de $\mathrm{Cu}-4 \mathrm{Ti}$. Rev. Metal. 46 (6), 542-547. http://dx.doi.org/10.3989/ revmetalmadrid. 1042 .

Donoso, E., Espinoza, R., Diánez, M.J., Criado, J.M. (2012). Microcalorimetric study of the annealing hardening mechanism of a Cu-2.8Ni-1.4Si (at\%) alloy. Mat. Sci. Eng. A-Struct. 556, 612-616. http://dx.doi.org/10.1016/j.msea. 2012.07.035.

Donoso, E. (2014). Estudio cinético de las reacciones de recocido en aleaciones de Cu-Ni-Fe. Rev. Metal. 50 (3), e020. http:// dx.doi.org/10.3989/revmetalm.020.

Donoso, E., Diánez, M.J., Criado J.M., Sayagués, M.J. (2014). Caracterización cinética de la precipitación en una aleación de $\mathrm{Cu}-11 \mathrm{Ni}-20 \mathrm{Zn}$ y $\mathrm{Cu}-12 \mathrm{Ni}-17 \mathrm{Zn}-2 \mathrm{Al}$. Congreso Internacional de Metalurgia y Materiales SAM-CONAMETIIBEROMATI MATERIA, Santa Fé, Argentina, pp. 1-4.

Gotor, F.J, Criado, J.M., Málek, J., Koga, N. (2000). Kinetic analysis of solid-state reactions: The universality of master plots for analyzing isothermal and nonisothermal experiments. J. Phys. Chem. A 104 (46), 10777-10782. http://dx.doi.org/ 10.1021/jp0022205.

Groza, J.R., Gibeling, J.C. (1993). Principles of particle selection for dispersion-strengthened copper. Mat. Sci. Eng. A-Struct. 171 (1-2), 115-125. http://dx.doi.org/10.1016/ 0921-5093(93)90398-X.

Hirabayashi, M., Hoshino, S., Sato, K. (1965). Neutron diffraction study of the long-range order in a single crystal $\mathrm{Cu}_{2}$ NiZn. J. Phys. Soc. Jpn. 20, 381-388. http://dx.doi. org/10.1143/JPSJ.20.381.

Kissinger, H.E. (1957). Reaction kinetics in Differential Thermal Analysis. Anal. Chem. 29 (11), 1702-1706. http://dx.doi. org/10.1021/ac60131a045.

Ito, T., Nakayama, Y. (1988). Dislocation-structures in deformed Cu-Ni-Zn alloy single-crystals. J. Mater. Sci. 23 (6), 2174-2180. http://dx.doi.org/10.1007/BF01115785.

Lebrun, N., Perrot, P. (2007). Non-ferrous Metal Ternary Systems: Selected Soldering and Brazing Systems, CopperNickel-Zinc, Vol. 11-C3, Effenberg G., Ilyenko S. (Eds.), Series Landolt-Börnstein-Group IV Physical Chemistry, Springer, Alemania, pp. 338-354.

Luo, Y.R. (2015). Handbook of Chemistry and Physics, Bond Dissociation Energies, $96^{\text {th }}$ Ed., Haynes W.M. Editor, CRC Press/Taylor and Francis, Boca Raton, Florida, pp. $9-65$.

Mittemeijer, E.J., Cheng, L., Van der Schaaf, P.J., Brakmany, C.M., Korevaar, B.M. (1988). Analysis of nonisothermal transformation kinetics; tempering of iron-carbon and iron-nitrogen martensites. Metall. Trans. A 19 (4), 925-932. http://dx.doi.org/10.1007/BF02628377.

Nagarjuna, S., Srinivas, M., Sharma, K.K. (2000). The grain size dependence of flow stress in a $\mathrm{Cu}-26 \mathrm{Ni}-17 \mathrm{Zn}$ alloy. Acta Mater. 48 (8), 1807-1813. http://dx.doi.org/10.1016/ S1359-6454(99)00464-4.

Nagarjuna, S., Gopalakrishna, B., Srinivas, M. (2006). On the strain hardening exponent of Cu-26Ni-17Zn alloy. Mat. Sci. Eng. A-Struct. 429 (1-2), 169-172. http://dx.doi.org/ 10.1016/j.msea.2006.05.096.

Ozawa, T. (1976). A modified method for kinetic analysis of thermoanalytical data. J. Therm. Anal. 9 (3), 369-373. http://dx.doi.org/10.1007/BF01909401.

Palma, R.H., Sepúlveda, A.O., Espinoza, R.G, Zúñiga, A.P., Diánez, M.J., Criado, J.M., Sayagués, M.J. (2004). Hightemperature mechanical behaviour of $\mathrm{Cu}-\mathrm{Ti}-\mathrm{C}, \mathrm{Cu}-\mathrm{Al}$ and $\mathrm{Cu}-\mathrm{Ti}-\mathrm{Al}-\mathrm{C}$ alloys obtained by reaction milling. Mat. Sci. Eng. A-Struct. 384 (1-2), 262-269. http://dx.doi.org/ 10.1016/j.msea.2004.06.036. 
Palma, R.H., Sepúlveda, A.O., Espinoza, R.G., Diánez, M.J., Criado, J.M., Sayagués, M.J. (2005). High-temperature deformation of dispersion-strengthened $\mathrm{Cu}-\mathrm{Zr}-\mathrm{Ti}-\mathrm{C}$ alloys. Mat. Sci. Eng. A-Struct. 391 (1-2), 60-65. http://dx.doi.org/ 10.1016/j.msea.2004.08.058.

Palma, R., Sepúlveda, A., Zúñiga, A., Donoso, E., Diánez, M.J., Criado, J.M. (2010). Microstructural characterization of copper based alloys produced by reactive milling. Rev. Metal. 46 (3), 197-205. http://dx.doi.org/10.3989/revmetalm.0854.

Pearson, W.B. (1958). A Handbook of Lattice Spacing and Structures of Metals and Alloys, Pergamon Press, pp. 571-574.

Simak, S.I., Ruban, A.V., Abrikosov, I.A., Skriver, H.L., Johansson, B. (1998). Ordered Phases in $\mathrm{Cu}_{2} \mathrm{NiZn}$ : A FirstPrinciples Monte Carlo Study. Phys. Rev. Lett. 81, 188-191. http://dx.doi.org/10.1103/PhysRevLett.81.188

Svadlak, Z. Zmirhalova, Z. Pustkova, P. Málek, J., Pérez-Maqueda, L.A., Criado, J.M. (2008). Crystallization behavior of $\left(\mathrm{GeS}_{2}\right) 0.1\left(\mathrm{Sb}_{2} \mathrm{~S}_{3}\right)_{0.9}$ glass. J. Non Cryst. Solids 354 (28), 3354-3361. http://dx.doi.org/10.1016/j.jnoncrysol. 2008.01.029.

Van der Wegen, G.J.L., De Rooy, A., Bronsveld, P.M., De Hosson, J.T.M. (1981). The order-didorder transition in the quasibinary cross section $\mathrm{Cu}_{50} \mathrm{Ni}_{50-\mathrm{x}} \mathrm{Zn}_{\mathrm{x}}$. I. Transmision Electron Microscopic Observations. Scripta Metall. 15, 1359-1364.
Van der Wegen, G.J.L., Helmholdt, R., Bronsweld, P., De Hosson, J. (1983). Single Crystal Neutron Diffraction Study of the Long-Range order in $\mathrm{Cu}_{2} \mathrm{NiZn}$. Z. Metallkd. 74 (9), 592-597.

Varschavsky, A., Donoso, E. (1991). Short range ordering by excess and thermal vacancies during linear heating experiments in $\alpha-\mathrm{Cu}-\mathrm{Al}$ alloys. Mat. Sci. Eng. A-Struct. 145 (1), 95-107. http://dx.doi.org/10.1016/0921-5093(91)90299-3.

Varschavsky, A., Donoso, E. (2002). Energetic and kinetic evaluations conducted in a quasi-binary $\mathrm{Cu}-1$ at. $\% \mathrm{Co}_{2} \mathrm{Si}$ alloy through DSC. J. Therm. Anal. Calorim. 68 (1), 231-241. http://dx.doi.org/10.1023/A:1014969618372.

Varschavsky, A., Donoso, E. (2003). Energetic and kinetic evaluations in a quasi-binary $\mathrm{Cu}-1$ at. $\% \mathrm{Co}_{2} \mathrm{Si}$. Mater. Lett. 57 (7), 1266-1271. http://dx.doi.org/10.1016/S0167-577X (02)00970-9.

Wnuk, G., Zielinska, M. (2009). Microstructural and thermal analysis of $\mathrm{Cu}-\mathrm{Ni}-\mathrm{Sn}-\mathrm{Zn}$ alloys by means of SEM and DSC techniques. Archives Mater. Sci. Eng. 40 (1), $27-32$.

Zhou, X.Z., Su, Y.C., Sun, J.M. (2010). Effect of aluminium on precipitation hardening in $\mathrm{Cu}-\mathrm{Ni}-\mathrm{Zn}$ alloys. $J$. Mater. Sci. 45 (11), 3080-3087. http://dx.doi.org/10.1007/s10853$010-4315-9$ 